\title{
Réforme éducative et cultures régionales en Russie
}

\section{Martine Faure}

\section{OpenEdition}

\section{Journals}

Édition électronique

URL : http://journals.openedition.org/ries/3329

DOI : $10.4000 /$ ries.3329

ISSN : 2261-4265

\section{Éditeur}

Centre international d'études pédagogiques

\section{Édition imprimée}

Date de publication : 1 juin 1996

Pagination : 103-109

ISSN : 1254-4590

\section{Référence électronique}

Martine Faure, "Réforme éducative et cultures régionales en Russie », Revue internationale d'éducation de Sèvres [En ligne], 10 | 1996, mis en ligne le 30 juillet 2013, consulté le 10 décembre 2020. URL http://journals.openedition.org/ries/3329 ; DOl : https://doi.org/10.4000/ries.3329

Ce document a été généré automatiquement le 10 décembre 2020.

() Tous droits réservés 


\title{
Réforme éducative et cultures régionales en Russie
}

\author{
Martine Faure
}

\section{Le nouveau cadre de l'école russe}

1 Après une longue période d'immobilisme, l'école russe a connu ces dernières années un profond bouleversement, commencé en 1985 avec la perestroika et s'accélérant au rythme des changements sociaux et économiques du pays. La politique de décentralisation, lancée dès 1991, commence à porter ses fruits dans les zones reculées de la Fédération de Russie, contribuant à faire évoluer l'offre éducative. Les nouveaux dispositifs s'éloignent du modèle soviétique, s'inspirant à la fois de systèmes étrangers et de la tradition de l'époque tsariste, tout en affichant la volonté d'une valorisation des patrimoines régionaux et nationaux.

2 Les changements ont été inscrits depuis la fin de l'URSS dans deux textes de loi successifs. La loi de juillet 1992 pose les jalons de la nouvelle école démocratique russe, réaffirme la nécessité d'une diversification des établissements déjà commencée pendant la perestroika et met fin au monopole d'État en matière d'éducation en permettant aux quatre-vingtneuf membres constitutifs de la Fédération de disposer d'un certain nombre de prérogatives dans le domaine de l'éducation. Appelés encore «entités de rang 2 », les membres de la nouvelle Fédération représentent des territoires très disparates, puisqu'il s'agit aussi bien des deux grandes villes de Moscou et de Saint-Pétersbourg, des vingt et une Républiques (comme la Carélie, la République des Tchouvaches ou le Tatarstan), des soixante-six unités régionales comportant entre elles des différences notables (les régions de la Russie centrale diffèrent par exemple profondément des territoires aux marges de l'ancien empire, comme le Kouban entre mer Noire et Caucase).

Quant à la récente loi de janvier 1996, elle permet notamment à la décentralisation de faire un pas de plus en conférant à l'échelon municipal la possibilité de concevoir une véritable politique scolaire et de gérer en direct les dépenses éducatives, par délégation de l'entité de rang 2. La loi autorise l'autogestion, en favorisant le principe de multi- 
financements par différents canaux et à différents niveaux de compétence. Les entités territoriales disposent ainsi de possibilités accrues pour lever des fonds complémentaires en faveur de l'éducation.

4 Une telle politique comporte quelques risques, notamment celui de creuser les différences déjà importantes entre régions riches et régions pauvres. Les régions puissantes, comme celle de Nijni-Novgorod ou d'Ekaterinbourg, sans compter Moscou et Saint-Pétersbourg, peuvent ainsi mener une action cohérente, les acquis de la métropole régionale profitant aux petites et moyennes villes. Des projets de développement se mettent en place avec succès à l'échelon régional et municipal, propres à créer une dynamique locale dans la population et à attirer l'attention de la société civile, qui soutient à son tour financièrement la création de nouvelles filières. Ailleurs, dans une situation où de nombreux établissements ne disposent pas du minimum nécessaire pour maintenir les bâtiments en état, l'urgence du quotidien tient lieu de projet pédagogique.

5 Le rôle de l'État, dans cette nouvelle donne, consiste à impulser le changement tout en assurant à chaque citoyen le droit à l'éducation et un minimum de garanties pour assurer la mobilité des cadres. Après une période de laisser-faire, les autorités éducatives centrales veillent au maintien de l'espace éducatif commun, comme le rappelle d'ailleurs explicitement la loi de 1996.

\section{L'école rurale et la décentralisation}

6 Actuellement, le développement de l'école rurale reste un des sujets de préoccupation du ministère de l'Éducation. Sur un plan qualitatif, l'éducation en zone rurale est restée en marge de la rénovation scolaire. Les établissements scolaires en zone urbaine ont été dynamisés par le passage à l'économie de marché et ses conséquences : certains secteurs créateurs de nouveaux emplois (banques, nouvelles entreprises de services...) sponsorisent en partie les innovations pédagogiques. Le fossé se creuse ainsi avec l'école rurale, car les zones agricoles, ne disposant pas des ressources naturelles propres à susciter les échanges commerciaux, restent à l'écart de ces évolutions et retiennent avec difficulté les jeunes au village. L'école rurale dispose de peu de moyens, d'autant plus qu'elle est faiblement dotée en raison de ses effectifs réduits.

7 Les autorités éducatives à l'échelon fédéral ont, dès 1991, encouragé les initiatives isolées d'institutions scolaires qui souhaitaient développer des mouvements alternatifs en éducation. Cependant, ces initiatives, qui ont surtout profité aux zones proches de la capitale, puis aux grandes villes de province, ont entraîné la création d'un secteur privé hautement sélectif, où sont scolarisés les enfants de l'intelligentsia et de la nouvelle classe socioprofessionnelle des « nouveaux Russes ».

8 Aujourd'hui, les pouvoirs publics cherchent à susciter une réflexion institutionnelle qui soit spécifique à l'école rurale, où l'on ne peut appliquer les solutions fondées sur une sélection sévère dans des zones fortement peuplées. Il s'agit maintenant de diffuser les résultats d'une recherche pédagogique dynamisée au service de terrains présentant pour le pédagogue chercheur des données stimulantes : habitat dispersé, diversité culturelle et ethnique, renouveau des écoles nationales, pédagogie des faibles effectifs ${ }^{1}$.

9 Sur un plan quantitatif, l'école rurale représente en effet la grande masse de la population scolaire, puisqu'il s'agit, selon les chiffres officiels du ministère de l'Éducation, de $80 \%$ des écoles russes. Les établissements d'un nouveau type, gymnases et lycées, ne sont 
présents en zones agricoles qu'à raison de $2 \%$ des écoles, alors que certaines grandes villes affirment que $25 \%$ de leurs établissements appartiennent à cette catégorie.

De plus, la dévalorisation actuelle de la fonction enseignante détourne les diplômés des instituts pédagogiques de l'enseignement en général et des écoles rurales en particulier. Actuellement, 30 \% seulement des jeunes diplômés choisissent une carrière enseignante et, parmi eux, la plupart cherchent à rester dans la capitale régionale où l'institut est luimême implanté. La suppression des affectations obligatoires après le diplôme entraîne une dotation insuffisante des villages en enseignants qualifiés; le faible niveau des salaires et le manque de ressources pédagogiques et culturelles des zones rurales ne permettent pas aux écoles reculées de susciter les vocations. Certaines disciplines sont particulièrement déficitaires, comme les mathématiques ou les langues étrangères, les nouveaux diplômés cherchant à s'installer dans des zones où les investissements étrangers leur permettront de compléter leurs salaires dans le secteur du commerce et des services, jusqu'à dix fois plus rémunérateur.

11 Malgré ces difficultés, l'examen de quelques tendances actuelles de la province montre que depuis 1990, l'école est devenue un large espace d'innovation, sur le plan administratif, pédagogique et culturel, dans lequel des autorités locales ont pu mener une politique de développement propre à leur zone et affirmer des options identitaires. Le ministère de l'Éducation de la République de Carélie, par exemple, a très tôt coopéré avec le ministère fédéral de l'Éducation pour expérimenter l'autonomie gestionnaire, ainsi que le Kouban ou la région de Barnaul dans l'Altaï. Dans ces trois exemples, les autorités éducatives sont portées par des courants identitaires forts. Le renouveau de la langue carélienne, proche du finnois, est conforté par les échanges avec la Finlande voisine ; les milieux cosaques du Kouban s'organisent et retrouvent leurs traditions ; la communauté d'origine allemande de l'Altaï a soutenu la création d'une université russo-allemande à Barnaul. Un tel mouvement prend souvent ses racines à la campagne, où les traditions et la langue ont été le mieux conservées, même s'il est ensuite relayé par l'intelligentsia urbaine. Certaines républiques riches s'appuient sur la rénovation du système scolaire pour mener une politique offensive de formation de cadres. Forte des ressources de son sous-sol, la Yakoutie sort de décennies d'acculturation en consacrant $40 \%$ de son budget à l'éducation. Le Tatarstan, quant à lui, mène une politique linguistique intensive, développe ses écoles nationales et forme dans les institutions de l'enseignement supérieur de Kazan des cadres pédagogiques aptes à enseigner en langue tatare: des enseignants de nationalité tatare résidant hors des frontières de la République peuvent également s'y former.

Plus généralement, l'école rurale, bien que peu touchée jusque là par la modernité ou manquant de moyens de mise en œuvre, dispose d'une réglementation mieux adaptée aux conditions spécifiques de scolarisation dans les zones isolées ou périphériques. Grâce à la décentralisation, les autorités régionales rénovent les programmes de formation continue des maîtres des anciens IPK (instituts de perfectionnement), et prennent en compte les besoins spécifiques des enseignants des zones éloignées. La nouvelle loi de 1996 permettra une véritable gestion municipale de l'éducation, plus proche des besoins des établissements. Dans les instituts pédagogiques relevant de l'État, l'innovation en zone rurale est également encouragée et de nouveaux cours sont consacrés à la pédagogie spécifique des écoles à petits effectifs ${ }^{2}$. 


\section{L'abandon du modèle universel}

13 Le passage d'une école soviétique à une école russe profitera-t-il aux zones rurales ? Le modèle précédent se voulait universel et égalitaire; il comportait un tronc unique dans le secondaire, puis des filières souvent très spécialisées dans le supérieur. La finalité de l'école soviétique était de former des citoyens au service d'une collectivité et d'une certaine vision du monde, créant un rapport spécifique à la science et à la nature. Aujourd'hui, l'objectif de la loi sur l'éducation est de permettre aux communautés de s'organiser elles-mêmes pour développer les ressources humaines locales et donner à chacun la possibilité de s'insérer dans la vie active en harmonie avec sa communauté d'appartenance, son territoire de résidence et les orientations fédérales. Les disparités régionales ne sont plus gommées dans les programmes, elles sont inscrites au contraire dans la législation, chaque membre de la Fédération pouvant passer un accord de coopération avec le gouvernement. Ce mouvement s'est rapidement développé, puisqu'aujourd'hui 70 membres sur 89 ont déjà signé un tel accord État/région, précisant les conditions d'un développement conjoint de la politique éducative.

Cette évolution s'est faite à la fois sur les finalités (quel lien entre école et profession, fondé sur quel lien entre individu et société), mais aussi sur les contenus (dépolitisation, désoviétisation et retour à une école nationale, russe ou autre) et enfin sur l'approche pédagogique (centration sur l'élève, individualisation des cursus). Pour l'instant, le principe démocratique du libre choix, associé à la diversification, a amené la multiplication des écoles à profils et, au sein des établissements, les classes à option. Dans les zones moins peuplées, on ne pourra sortir du système du tronc unique de neuf années que par la pédagogie différenciée, permettant de proposer à chaque élève un parcours qui lui soit adapté. Or, si le concept de centration sur l'élève apparaît maintenant dans les discours des nouveaux méthodologues, son application sur le terrain par les enseignants reste à mettre en œuvre.

La longueur du chemin à parcourir sera fortement liée à la compréhension complète du dernier principe, celui de dépolitisation. Le modèle soviétique affirmait la suprématie du groupe sur l'individu et cloisonnait les catégories; il faut donc créer le mouvement inverse en privilégiant des méthodes d'individualisation, de prise d'autonomie et d'intégration horizontale. Or les campagnes sont en retard dans l'obtention de nouveaux matériels pédagogiques dépolitisés et disposent de peu de ressources culturelles dans l'environnement immédiat de l'école. Il faut avant tout changer les mentalités des enseignants et les aider à créer des ressources éducatives issues du patrimoine local. Certains instituts pédagogiques développent des cours spécifiques, consacrés à la redécouverte et l'exploitation pédagogique du patrimoine, en liaison avec la politique régionale culturelle.

\section{Diversité et développement local}

\section{Innovations pédagogiques}

Une tendance forte pour encourager le processus de rénovation scolaire est d'augmenter la polyvalence des enseignants, en la valorisant par d'autres pratiques pédagogiques. Tout en apportant un début de solution au déficit d'enseignants et au problème des faibles 
effectifs, cette approche permet de décloisonner les disciplines en travaillant de façon transversale sur un thème donné. Dans un récent colloque sur l'école rurale, le ministre de l'éducation Tkatchenko incitait les enseignants à innover dans ce secteur et se félicitait du travail déjà engagé dans la formation des enseignants. Des séminaires locaux dynamisent le tissu pédagogique des petites villes et encouragent les échanges et la création d'associations professionnelles propres à rompre l'isolement matériel et surtout moral de ces enseignants. Enfin, les milieux pédagogiques s'expriment davantage à travers des associations et leurs journaux professionnels, qu'ils soient rénovés, comme Outchitielskaya gazieta, ou créés après la fin du régime soviétique, comme Piervoïe sientiabria ou le récent Direktor chkoly. Les débats et l'abondant courrier des lecteurs créent le lien pour des acteurs isolés.

Un fort encouragement a été donné à la réalisation de supports pédagogiques. Autrefois réservée à des méthodologues appointés par l'État, l'élaboration et l'édition de manuels se situent désormais sur un marché concurrentiel. Le ministère de l'Éducation, avec l'appui de la Fondation Soros, a lancé un concours de manuels s'adressant à tous les enseignants. Un accent prioritaire a été mis sur les écoles ordinaires, pour servir la grande masse des écoles de petites villes et de villages, où le manuel de base reste l'outil de référence.

Des organisations non gouvernementales se spécialisent par ailleurs dans le conseil en politique scolaire : le mouvement pédagogique «Evrika», de l'époque de la perestroika, bien implanté dans les régions périphériques, a permis par exemple l'éclosion de plusieurs associations ou cabinets d'études spécialisés dans les politiques scolaires; progressivement ces mouvements ont compris la nécessité de traiter la question scolaire dans le cadre du développement local. Des experts individuels, des organismes comme le MIROS (Centre moscovite de développement des systèmes éducatifs) interviennent à la demande des pouvoirs locaux pour mettre en place des plans de restructuration ou d'accompagnement scolaire.

\section{Réseaux et communication}

Une nouvelle demande s'exprime dans les zones de peuplement pionnier: les petites villes créées pour les besoins de l'extraction et de l'exploitation des ressources naturelles, par exemple sur des sites miniers, pétrolifères ou nucléaires. Les familles vivant dans ces villes, attirées par les fortes primes, n'avaient en général pas le projet de s'y installer de façon durable. Les bouleversements politiques et économiques, et l'inflation galopante qui en a découlé, ont soit réduit des économies à néant, soit beaucoup limité le pouvoir d'achat et de déplacement de ces personnes. Aussi les familles sont-elles amenées à scolariser sur place les jeunes, et se tournent-elles vers les autorités locales avec de nouvelles exigences: créer sur place un dispositif cohérent, diversifié et non lacunaire malgré les faibles effectifs. Plusieurs études actuellement en cours, sur des commandes institutionnelles, se fondent sur la nécessité d'une démarche intégrée: s'aider de l'environnement pour développer des ressources éducatives, aider par la formation l'environnement à se structurer et s'enrichir ; car le problème de ces petites villes ne se situe pas tant dans les moyens que dans l'isolement.

Pour des cas comme ceux-là, la mise en réseau par la formation à distance et l'enseignement ouvert, le courrier électronique, les nouvelles technologies en général sont des moyens adaptés à la population formée principalement de cadres de culture scientifique et technique. De tels réseaux commencent à se développer, avec le soutien 
notamment de la Commission européenne (citons, par exemple, la création du réseau LINK dans le cadre du programme TACIS).

21 La configuration géographique éclatée de la Russie, les disparités économiques régionales, l'existence d'une importante diversité ethnique sont autant de facteurs contribuant à la prise en compte des identités locales dans le système éducatif. La grande presse elle-même se fait également l'écho du débat scolaire. En septembre 1995, le journal Argumenty i fakty citait une manifestation intéressante : l'association des enseignants pour le renouveau des écoles de zemstvos ${ }^{3}$ 'était réunie pour examiner la possibilité d'un retour à l'auto-gestion de l'éducation.

L'aspiration à une école plus proche des habitants et réinvestie par ses acteurs semble bien correspondre à une lame de fond de la société et des professionnels de l'éducation. Qu'elle soit portée par une nostalgie du passé, par une confrontation avec certains modèles occidentaux fortement décentralisés ou par des communautés soudées autour de leur patrimoine linguistique et culturel, la gestion locale de l'éducation semble largement approuvée par la demande sociale. L'État russe cherche aujourd'hui un équilibre entre la limitation des débordements ethniques et religieux, et l'encouragement à l'expression régionale et nationale pour éviter le double isolement de certaines communautés et le risque d'éclatement de l'espace éducatif commun. Il est trop tôt pour dire si la loi de janvier 1996 portera tous ces effets, mais des expériences encourageantes démontrent qu'elle constitue un grand pas en avant dans le processus de réforme engagé depuis l'effondrement de l'URSS.

\section{NOTES}

1. Voir les travaux de Sirioulnikov, de l'Institut de recherche sur l'éducation régionale et de l'Institut de perfectionnement des cadres de l'éducation, le RIPKRO.

2. Voir par exemple les expériences en zones agraires de la Russie centrale comme Koursk, Orel.

3. Les zemstvos étaient, à l'époque tsariste, des assemblées élues provinciales qui avaient en charge certains secteurs de proximité comme l'éducation, mais aussi la santé et les transports.

\section{RÉSUMÉS}

Conséquence des changements sociaux et économiques intervenus en Russie, le processus de décentralisation s'inscrit dans la nouvelle loi sur l'éducation de 1996, qui accorde aux communes les compétences permettant à l'école rurale de s'organiser et de devenir partie prenante dans un schéma de développement local. 
INDEX

Mots-clés : décentralisation, développement rural, école rurale, réforme, système éducatif Index géographique : Russie

\section{AUTEUR}

MARTINE FAURE

Chargée d'études, département des systèmes éducatifs, CIEP, France. 